\title{
A Tuning Procedure for ARX-based MPC of Multivariate Processes
}

\author{
Olesen, Daniel; Huusom, Jakob Kjøbsted; Jørgensen, John Bagterp
}

Published in:

2013 American Control Conference (ACC)

Publication date:

2013

Document Version

Publisher's PDF, also known as Version of record

Link back to DTU Orbit

Citation (APA):

Olesen, D., Huusom, J. K., \& Jørgensen, J. B. (2013). A Tuning Procedure for ARX-based MPC of Multivariate Processes. In 2013 American Control Conference (ACC) (pp. 1721-1726). IEEE. American Control Conference

\section{General rights}

Copyright and moral rights for the publications made accessible in the public portal are retained by the authors and/or other copyright owners and it is a condition of accessing publications that users recognise and abide by the legal requirements associated with these rights.

- Users may download and print one copy of any publication from the public portal for the purpose of private study or research.

- You may not further distribute the material or use it for any profit-making activity or commercial gain

- You may freely distribute the URL identifying the publication in the public portal

If you believe that this document breaches copyright please contact us providing details, and we will remove access to the work immediately and investigate your claim. 


\title{
A Tuning Procedure for ARX-based MPC of Multivariate Processes
}

\author{
Daniel Haugaard Olesen, Jakob Kjøbsted Huusom, John Bagterp Jørgensen
}

\begin{abstract}
We present an optimization based tuning procedure with certain robustness properties for an offset free Model Predictive Controller (MPC). The MPC is designed for multivariate processes that can be represented by an ARX model. The stochastic model of the ARX model identified from inputoutput data is modified with an ARMA model designed as part of the MPC-design procedure to ensure offset-free control. The MPC is designed and implemented based on a state space model in innovation form. Expressions for the closed-loop dynamics of the unconstrained system is used to derive the sensitivity function of this system. The closed-loop expressions are also used to numerically evaluate absolute integral performance measures. Due to the closed-loop expressions these evaluations can be done relative quickly. Consequently, the tuning may be performed by numerical minimization of the integrated absolute error subject to a constraint on the maximum of the sensitivity function. The latter constraint provides a robustness measure that is essential for the procedure. The method is demonstrated for two simulated examples: A Wood-Berry distillation column example and a cement mill example.
\end{abstract}

\section{INTRODUCTION}

Model Predictive Control (MPC) has evolved to become an industrial standard in advanced process control [1]. Using a model of the system to predict the process output over some future horizon, MPC computes a trajectory of manipulated inputs such that the predicted future output is as desirable as possible. Only the inputs related to the first period in this trajectory are implemented. As new measurements become available, the estimation and regulation windows are shifted and the estimation and optimization procedures are repeated. In this paper we consider MPC based on ARX models. An ARX model representation of the plant may be obtained from input-output data using convex optimization methods [2]. To ensure offset free control, integrators have to be introduced in the plant model in case of persistent unmeasured disturbances and/or plant model mismatch. In such cases, the observer that guarantees offset free control introduces a plant model mismatch. This plant model mismatch complicates the tuning of the controller [3]-[6].

Despite the growing popularity of MPC, a systematic tuning practice has not evolved, and only few guidelines exist. The topic has not been short of research, as there are numerous academic publications on the subject. A comprehensive review of proposed tuning methods is presented by [7] and loop transfer recovery procedures have also been investigated [8], [9]. Our study relies on a closed loop description of the

D.H. Olesen and J.B. Jørgensen are with DTU Compute, Technical University of Denmark, DK-2800 Kgs Lyngby, Denmark. Corresponding author: J.B. Jørgensen, jb jo@dtu. dk

J.K. Huusom is with DTU Chemical Engineering, Technical University of Denmark, DK-2800 Kgs Lyngby, Denmark controller and the process model to assess the performance of an MPC with a given tuning. It has previously been proposed to use a closed loop description for synthesis of a MPC by application of robust design techniques [10]. In this paper, we state the tuning problem as an inequality constrained optimization problem. We propose a deterministic tuning objective function related to the integrated absolute error for a number of pre-defined scenarios and use a bound on the maximum sensitivity to ensure robustness.

The paper is organized as follows. Section II describes an ARX-based MPC for multivariate processes. Section III derives a state-space model for the closed-loop system and uses this state space model for covariance computation and sensitivity function computation. IAE measures and the sensitivity function are used to formulate an optimization problem for selecting the tuning parameters of the MPC. Section IV demonstrates the procedure for a Wood-Berry binary distillation example, while Section $\mathrm{V}$ provides a case study for a simulated cement mill. Conclusions are presented in Section VI.

\section{ARX-BASED MPC FOR MIMO SYSTEMS}

In this section, we derive a state space representation for an unconstrained MPC based on MISO ARX-models modified with a filtered integrated white noise stochastic model. First, we represent the MISO ARX model as a state space model in innovation form. Subsequently, we use this state space model in innovation form to derive the correct control law for the unconstrained MPC. As the control law is linear the resulting controller may be represented in a state space form.

\section{A. State Space Model in Innovation Form}

The MISO ARX model

$$
A_{i}\left(q^{-1}\right) y_{i, k}=B_{i}\left(q^{-1}\right) u_{k}+\varepsilon_{i, k} \quad i=1, \ldots, n_{y}
$$

with $y_{i, k} \in \mathbb{R}$ for $i=1, \ldots, n_{y}, u_{k} \in \mathbb{R}^{n_{u}}$, and $\varepsilon_{i, k} \in$ $\mathbb{R}$ for $i=1, \ldots, n_{y}$ has been used in a number of MPC applications. The advantage of this model parametrization is that the parameters may be identified using standard system identification techniques based on convex optimization. To have offset-free control from the MPC based on this model, the stochastic part of the model is modified to be a filtered white noise process

$$
\varepsilon_{i, k}=\frac{1-\alpha_{i} q^{-1}}{1-q^{-1}} e_{i, k} \quad i=1, \ldots, n_{y}
$$

where $e_{i, k} \sim \mathcal{N}_{i i d}\left(0, R_{e e}\right)$. The coefficients $\alpha_{i}$ are design parameters of the MPC. 
The representation of the MIMO system from these MISO systems is not unique. One straightforward representation leading to a compact notation is

$$
A\left(q^{-1}\right) y_{k}=B\left(q^{-1}\right) u_{k}+\left(I-I q^{-1}\right)^{-1}\left(I-\mathcal{A} q^{-1}\right) e_{k}
$$

with $A\left(q^{-1}\right)=\operatorname{diag}\left(\left[A_{1}\left(q^{-1}\right) ; \ldots ; A_{n_{y}}\left(q^{-1}\right)\right]\right), B\left(q^{-1}\right)=$ $\left[B_{1}\left(q^{-1}\right) ; \ldots ; B_{n_{y}}\left(q^{-1}\right)\right]$, and $\mathcal{A}=\operatorname{diag}\left(\left[\alpha_{1} ; \ldots ; \alpha_{n_{y}}\right]\right)$. This model can be represented as an ARMAX model

$$
\bar{A}\left(q^{-1}\right) y_{k}=\bar{B}\left(q^{-1}\right) u_{k}+\bar{C}\left(q^{-1}\right) e_{k}
$$

with

$$
\begin{aligned}
& \bar{A}\left(q^{-1}\right)=\left(I-I q^{-1}\right) A\left(q^{-1}\right) \\
& \bar{B}\left(q^{-1}\right)=\left(I-I q^{-1}\right) B\left(q^{-1}\right) \\
& \bar{C}\left(q^{-1}\right)=I-\mathcal{A} q^{-1}
\end{aligned}
$$

Denote the coefficients of $\bar{A}\left(q^{-1}\right)$ and $\bar{B}\left(q^{-1}\right)$ as

$$
\begin{aligned}
& \bar{A}\left(q^{-1}\right)=I+\bar{A}_{1} q^{-1}+\bar{A}_{2} q^{-2}+\ldots+\bar{A}_{n} q^{-n} \\
& \bar{B}\left(q^{-1}\right)=\bar{B}_{1} q^{-1}+\bar{B}_{2} q^{-2}+\ldots+\bar{B}_{n} q^{-n}
\end{aligned}
$$

Then the system (1)-(2) may be represented as a state space model in innovation form

$$
\begin{aligned}
x_{k+1} & =\hat{A} x_{k}+\hat{B} u_{k}+\hat{K} e_{k} \\
y_{k} & =\hat{C} x_{k}+e_{k}
\end{aligned}
$$

with the state space matrices $(\hat{A}, \hat{B}, \hat{K}, \hat{C})$ realized in observer canonical form

$$
\begin{aligned}
\hat{A} & =\left[\begin{array}{c|cccc}
-\bar{A}_{1} & I & 0 & 0 & 0 \\
-\bar{A}_{2} & 0 & I & 0 & 0 \\
\vdots & \vdots & & \ddots & \vdots \\
-\bar{A}_{n-1} & 0 & 0 & \cdots & I \\
\hline-A_{n} & 0 & 0 & \cdots & 0
\end{array}\right] \hat{B}=\left[\begin{array}{c}
\bar{B}_{1} \\
\bar{B}_{2} \\
\vdots \\
\bar{B}_{n-1} \\
\bar{B}_{n}
\end{array}\right] \hat{K}=\left[\begin{array}{c}
\mathcal{A}-\bar{A}_{1} \\
-\bar{A}_{2} \\
\vdots \\
-\bar{A}_{n-1} \\
-\bar{A}_{n}
\end{array}\right] \\
\hat{C} & =\left[\begin{array}{lllll}
I & 0 & 0 & \cdots & 0
\end{array}\right]
\end{aligned}
$$

\section{B. Unconstrained MPC for State Space Models in Innova- tion Form}

The filtered state estimation and the one-step prediction may for state space models in innovation form (7) be combined to give the following expressions for computation of the innovation, $e_{k}$ [5]:

$$
\begin{aligned}
\hat{x}_{k \mid k-1} & =\hat{A} \hat{x}_{k-1 \mid k-2}+\hat{B} u_{k-1}+\hat{K} e_{k-1} \\
\hat{y}_{k \mid k-1} & =\hat{C} \hat{x}_{k \mid k-1} \\
e_{k} & =y_{k}-\hat{y}_{k \mid k-1}
\end{aligned}
$$

Initially, $\hat{x}_{0 \mid-1}$ is known and the one-step prediction (8a) is not needed. Knowing the innovation, $e_{k}$, the predictions in the state space model in innovation form may be represented as [5]

$$
\begin{aligned}
\hat{x}_{k+1 \mid k} & =\hat{A} \hat{x}_{k \mid k-1}+\hat{B} \hat{u}_{k \mid k}+\hat{K} e_{k} \\
\hat{x}_{k+1+j \mid k} & =\hat{A} \hat{x}_{k+j \mid k}+\hat{B} \hat{u}_{k+j \mid k}, j=1, \ldots, N-1 \\
\hat{y}_{k+j \mid k} & =\hat{C} \hat{x}_{k+j \mid k}, j=1, \ldots, N
\end{aligned}
$$

It is important to notice the term $\hat{K} e_{k}$ in (9a). This term is important for derivation of the correct control law [5]. Let the objective of the MPC be

$$
\phi=\frac{1}{2} \sum_{j=0}^{N-1}\left\|\hat{y}_{k+j+1 \mid k}-r_{k+j+1 \mid k}\right\|_{Q}^{2}+\left\|\Delta \hat{u}_{k+j \mid k}\right\|_{S}^{2}
$$

in which the second term, $\left\|\Delta \hat{u}_{k+j \mid j}\right\|_{S}^{2}$, is a regularization term. We assume the reference parametrization, $\left\{r_{k+j \mid k}\right\}_{j=1}^{N}=\left\{r_{k}, \ldots, r_{k}\right\}$. The tuning parameters in this objective function are the matrices $Q=\operatorname{diag}\left(\left[q_{1} ; \ldots ; q_{n_{y}}\right]\right)$ and $S=\operatorname{diag}\left(\left[s_{1} ; \ldots ; s_{n_{u}}\right]\right)$. As indicated, these matrices are restricted to diagonal matrices.

The unconstrained MPC may be represented as the convex quadratic optimization problem

$\min _{\left\{\hat{u}_{k+j \mid j}\right\}_{j=0}^{N-1}}\left\{\phi=\phi\left(\left\{\hat{u}_{k+j \mid j}\right\}_{j=0}^{N-1} ; \hat{x}_{k \mid k-1}, r_{k}, u_{k-1}, e_{k}\right):\right.$

which has the solution $U_{k}=\left[\hat{u}_{k \mid k}, \ldots, \hat{u}_{k+N-1 \mid k}\right]$ with [5]

$$
u_{k}=\hat{u}_{k \mid k}=L_{x} \hat{x}_{k \mid k-1}+L_{w} e_{k}+L_{u} u_{k-1}+L_{r} r_{k}
$$

The specific expressions for and derivation of $L_{x}, L_{w}, L_{u}$ and $L_{r}$ are given in [5]. It must be emphasized that most available expressions for linear-quadratic controllers misses the term $L_{w} e_{k}$ that arises due to the term $\hat{K} e_{k}$ in (9a).

Define the controller states as $x_{k}^{c}=\left[\hat{x}_{k \mid k-1} ; u_{k-1}\right]$ such that the unconstrained MPC consisting of (8) and (11) may be represented in the state space form

$$
\begin{aligned}
x_{k+1}^{c} & =A_{c} x_{k}^{c}+B_{c y} y_{k}+B_{c r} r_{k} \\
u_{k} & =C_{c} x_{k}^{c}+D_{c y} y_{k}+D_{c r} r_{k}
\end{aligned}
$$

with

$$
\begin{aligned}
A_{c} & =\left[\begin{array}{rr}
(\hat{A}-\hat{K} \hat{C})+\hat{B}\left(L_{x}-L_{w} \hat{C}\right) & \hat{B} L_{u} \\
L_{x}-L_{w} \hat{C} & L_{u}
\end{array}\right] \\
B_{c y} & =\left[\begin{array}{c}
\hat{K}+\hat{B} L_{w} \\
L_{w}
\end{array}\right] \quad B_{c r}=\left[\begin{array}{c}
\hat{B} L_{r} \\
L_{r}
\end{array}\right] \\
C_{c} & =\left[\begin{array}{ll}
L_{x}-L_{w} \hat{C} & L_{u}
\end{array}\right] \quad D_{c r}=L_{r} \\
D_{c y} & =L_{w}
\end{aligned}
$$

In addition to the model (1), this controller representation depends on the tuning parameters

$$
\begin{aligned}
\mathcal{A} & =\operatorname{diag}\left(\left[\alpha_{1} ; \ldots ; \alpha_{n_{y}}\right]\right) \\
Q & =\operatorname{diag}\left(\left[q_{1} ; \ldots ; q_{n_{y}}\right]\right) \\
S & =\operatorname{diag}\left(\left[s_{1} ; \ldots ; s_{n_{u}}\right]\right)
\end{aligned}
$$

\section{Closed-Loop System and Measures}

Let the system be a LTI system in the form

$$
\begin{aligned}
x_{k+1} & =A x_{k}+B u_{k}+E d_{k}+G w_{k} \\
z_{k} & =C x_{k} \\
y_{k} & =z_{k}+v_{k}
\end{aligned}
$$

where $x_{k}$ is states, $u_{k}$ is manipulated inputs, $d_{k}$ is unknown disturbances, $w_{k} \sim N_{i i d}\left(0, R_{w w}\right)$ is process noise, $z_{k}$ is outputs, $v_{k} \sim N_{i i d}\left(0, R_{v v}\right)$ is measurement noise, and $y_{k}$ is measurements, i.e. the outputs, $z_{k}$, corrupted by 


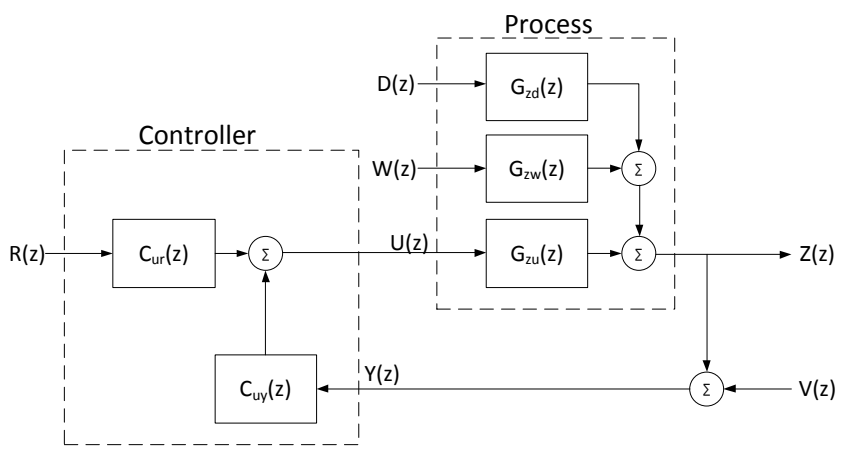

Fig. 1. Closed-loop system. The transfer functions $C_{u r}(z)$ and $C_{u y}(z)$ forms the unconstrained MPC. The controlled outputs of the process is described by $G_{z u}(z), G_{z w}(z)$ and $G_{z d}(z)$.

measurement noise, $v_{k}$. This model $(A, B, E, G, C)$ is not necessarily identical to the model $(\hat{A}, \hat{B}, \hat{C}, \hat{K})$ used by the MPC. Using the system model (15) and the MPC state space representation (12), the closed-loop system may be represented as

$$
\begin{aligned}
x_{k+1}^{c l} & =A_{c l} x_{k}^{c l}+B_{w c l} w_{k}+B_{v c l} v_{k}+B_{r c l} r_{k}+B_{d c l} d_{k} \\
z_{k} & =C_{c l} x_{k}^{c l} \\
y_{k} & =C_{c l} x_{k}^{c l}+v_{k} \\
u_{k} & =C_{u c l} x_{k}^{c l}+D_{v c l} v_{k}+D_{r c l} r_{k}
\end{aligned}
$$

with $x_{k}^{c l}=\left[x_{k} ; x_{k}^{c}\right]$ and

$$
\begin{aligned}
& A_{c l}=\left[\begin{array}{cc}
A+B D_{c y} C & B C_{c} \\
B_{c y} C & A_{c}
\end{array}\right], B_{w c l}=\left[\begin{array}{c}
G \\
0
\end{array}\right], \\
& B_{v c l}=\left[\begin{array}{c}
B D_{c y} \\
B_{c y}
\end{array}\right], B_{r c l}=\left[\begin{array}{c}
B D_{c r} \\
B_{c r}
\end{array}\right], B_{d c l}=\left[\begin{array}{c}
E \\
0
\end{array}\right], \\
& C_{c l}=\left[\begin{array}{ll}
C & 0
\end{array}\right], C_{u c l}=\left[\begin{array}{ll}
D_{c y} C & C_{c}
\end{array}\right], \\
& D_{v c l}=D_{c y}, D_{r c l}=D_{c r} .
\end{aligned}
$$

This representation depends on the MPC tuning parameters, $(\mathcal{A}, Q, S)$, and is used extensively to compute measures for the controller performance. An obvious measure is the eigenvalues of the closed-loop system, $\lambda=\operatorname{eig}\left(A_{c l}\right)$, as acceptable tunings must provide stable closed loop systems.

\section{A. Covariance}

The covariance of the outputs, $\left(z_{k}, y_{k}, u_{k}\right)$, for the closedloop system as response to the exogenous stochastic signals, $w_{k}$ and $v_{k}$, is one measure for the performance of the MPC.

Provided that $A_{c l}$ is stable, the covariance of the states of the closed loop system, $R_{x x}$, may be computed by solution of the discrete Lyapunov equation

$$
R_{x x}=A_{c l} R_{x x} A_{c l}^{T}+B_{w c l} R_{w w} B_{w c l}^{T}+B_{v c l} R_{v v} B_{v c l}^{T}
$$

The corresponding output covariances are

$$
\begin{aligned}
& R_{z z}=C_{c l} R_{x x} C_{c l}^{T} \\
& R_{y y}=C_{c l} R_{x x} C_{c l}^{T}+R_{v v} \\
& R_{u u}=C_{u c l} R_{x x} C_{u c}^{T}+D_{v c l} R_{v v} D_{v c l}^{T}
\end{aligned}
$$

\section{B. Sensitivity}

Fig. 1 illustrates the transfer functions in the process model and the model predictive controller. The transfer function model of the open-loop system (15) is

$$
Y(z)=G_{z u}(z) U(z)+G_{z d}(z) D(z)+G_{z w} W(z)+V(z)
$$

and the transfer function model of the MPC control law (12) may be represented as

$$
U(z)=C_{u y}(z) Y(z)+C_{u r}(z) R(z)
$$

$G_{z u}(z), G_{z d}(z), G_{z w}(z), C_{u y}(z)$, and $C_{u r}(z)$ may be computed from the associated state-space representations in the standard way. Combining (20) and (21) yields a transfer function for the closed-loop system (16)

$$
Y(z)=S(z) \bar{D}(z)+T(z) R(z)
$$

with $\bar{D}(z)=G_{z d}(z) D(z)+G_{z w}(z) W(z)+V(z)$ and

$$
\begin{aligned}
& S(z)=C_{c l}\left(z I-A_{c l}\right)^{-1} B_{v c l}+I \\
& T(z)=C_{c l}\left(z I-A_{c l}\right)^{-1} B_{r c l}
\end{aligned}
$$

$S(z)$ is the sensitivity function and $T(z)$ is the complementary sensitivity function. The sensitivity function, $S(z)$, is related to the robustness of the system in relation to modelplant mismatch as well as process and measurement noise [11]. In particular the $\mathcal{H}_{\infty}$ norm of $S(z)$

$$
M_{S}=\|S(z)\|_{\infty}=\max _{\omega} \bar{\sigma}\left(S\left(e^{j \omega T_{s}}\right)\right)
$$

has been used as a measure of robustness. $\bar{\sigma}$ denotes the maximum singular value and $T_{s}$ denotes the sampling time.

\section{Integrated Absolute Error}

The integrated absolute error (IAE) is a classical way to measure control systems performance for certain reference and disturbance scenarios of systems without noise $\left(w_{k}=\right.$ 0 and $v_{k}=0$ ). Consider a scenario starting from steady state and specified by $[r(t)]_{t_{0}}^{t_{f}}=\left\{r_{k}\right\}_{k=0}^{n_{f}-1}$ and $[d(t)]_{t_{0}}^{t_{f}}=$ $\left\{d_{k}\right\}_{k=0}^{n_{f}-1}$ with $r(t)=r_{k}$ and $d(t)=d_{k}$ for $t_{k} \leq t<t_{k+1}$ using $t_{k}=t_{0}+k T_{s}$ and $t_{f}=t_{0}+n_{f} T_{s}$. The IAE of this scenario is approximated by euler integration to be:

$$
J_{i}=\sum_{k=0}^{n_{f}-1}\left|y_{i, k}-r_{i, k}\right| \quad i=1, \ldots, n_{y}
$$

Equation (25) is evaluated by simulation using the deterministic part of (16), the initial steady state, $x_{0}^{c l}=0$, and the specified scenario, $\left\{r_{k}\right\}_{k=0}^{n_{f}-1}$ and $\left\{d_{k}\right\}_{k=0}^{n_{f}-1}$.

The scenarios, $j \in \mathcal{S}$, for evaluation of the IAE-measures, $J_{i j}$ with $i=1, \ldots, n_{y}$ can be chosen according to the tasks of a given control system. In this paper we consider two standard type of scenarios. The first type of scenarios are related to individual set-point changes and consist of a set of $n_{y}$ scenarios, $\mathcal{S}_{r}$, with unit step changes in each individual reference, $\left(r_{j}\right)_{k}=1$ for $0 \leq k<n_{f}$ and $j \in \mathcal{S}_{r}$. We denote the performance matrix associated with these scenarios $J_{r}=\left[J_{i j}\right]$ for $i=1, \ldots, n_{y}$ and $\forall j \in \mathcal{S}_{r}$. The second type of scenarios are related to disturbance rejection. 
This set of scenarios, $\mathcal{S}_{d}$, consists of $n_{d}$ scenarios with a unit step in each individual disturbance, $\left(d_{j}\right)_{k}=1$ for $0 \leq k<n_{f}$ and $j \in \mathcal{S}_{d}$. The performance matrix associated with these scenarios is denoted $J_{d}=\left[J_{i j}\right]$ with $i=1, \ldots, n_{y}$ and $j \in \mathcal{S}_{d}$.

\section{Tuning}

In the tuning of the MPC, the control and prediction horizon, $N$, is chosen sufficiently large such that the resulting controller for all practical purposes corresponds to an infinite horizon controller. The remaining tuning parameters, $(\mathcal{A}, Q, S)$, are chosen by solution of the constrained optimization problem

$$
\begin{array}{ll}
\min _{\mathcal{A}, Q, S} & J=\left\|J_{r}(\mathcal{A}, Q, S)\right\|_{2}+\left\|J_{d}(\mathcal{A}, Q, S)\right\|_{2} \\
\text { s.t. } & M_{S}(\mathcal{A}, Q, S) \leq M_{S, \max } \\
& 0 \leq \mathcal{A} \leq I \\
& 0 \leq Q \leq Q_{\max } \\
& 0 \leq S \leq S_{\max }
\end{array}
$$

The objective minimizes some measure related to the IAE of the chosen scenarios. In the cases studied in this paper, we have used the sum of the 2-norms of the matrices associated with the IAE of setpoint changes and disturbance rejections. One could also use the sum of all scenarios, $J=\sum_{j \in \mathcal{S}} \sum_{i=1}^{n_{y}} J_{i j}$ with $\mathcal{S}=\mathcal{S}_{r} \cup \mathcal{S}_{d}$, and expect similar results. It is critical for the usefulness of the resulting tuning that the robustness constraint (26b) is included in the optimization problem. Useless results are obtained if the robustness constraint (26b) is discarded by using a large upper bound. In such cases, the resulting controller is far too aggressive and useless in practice. $M_{S, \max }$ is a user selected parameter used for deciding how robust the resulting closed loop system should be. Smaller values gives a less aggressive and more robust controller.

Equation (26) is a constrained nonlinear optimization problem which is not necessarily convex. Accordingly, we cannot guarantee location of the global optimum of (26) when using solvers such as fmincon, KNITRO, IPOPT, NLOPT, or SNOPT.

\section{WOOD-BERRY Distillation COLUMN}

We consider a Wood-Berry binary distillation column that has the input-output description

$$
Y(s)=G_{u}(s) U(s)+G_{d}(s)(D(s)+W(s))+V(s)
$$

with $u(t)=u_{k}, d(t)=d_{k}$ and $w(t)=w_{k} \sim N_{i i d}\left(0, R_{w w}\right)$ being piecewise constant in the interval $t_{k} \leq t<t_{k+1}$ and $v\left(t_{k}\right)=v_{k} \sim N_{i i d}\left(0, R_{v v}\right)$. The transfer functions are [12]

$$
\begin{aligned}
G_{u}(s) & =\left[\begin{array}{ll}
\frac{12.8}{16.7 s+1} e^{-s} & \frac{-18.9}{21.0 s+1} e^{-3 s} \\
\frac{6.6}{10.9 s+1} e^{-7 s} & \frac{-19.4}{14.4 s+1} e^{-3 s}
\end{array}\right] \\
G_{d}(s) & =\left[\begin{array}{l}
\frac{3.8}{14.9 s+1} e^{-8.1 s} \\
\frac{4.9}{13.2 s+1} e^{-3.4 s}
\end{array}\right]
\end{aligned}
$$

\begin{tabular}{|c|c|c|}
\hline & Design & Simulation \\
\hline \multirow{2}{*}{$J_{r}$} & $\begin{array}{ll}9.78 & 1.78\end{array}$ & $\begin{array}{ll}11.14 & 3.19\end{array}$ \\
\hline & 12.08 & 13.45 \\
\hline \multirow{2}{*}{$J_{d}$} & \multirow{2}{*}{$\begin{array}{c}7.22 \\
20.27\end{array}$} & \multirow{2}{*}{$\begin{array}{l}8.403 \\
21.54\end{array}$} \\
\hline & & \\
\hline \multirow{2}{*}{$R_{y y}$} & $1.34 \cdot 10^{-4} \quad 0.19 \cdot 10^{-4}$ & $1.28 \cdot 10^{-4} \quad 0.14 \cdot 10^{-4}$ \\
\hline & $0.19 \cdot 10^{-4}$ & $0.14 \cdot 10^{-4}$ \\
\hline \multirow{2}{*}{$R_{u u}$} & $9.26 \cdot 10^{-6}$ & $8.93 \cdot 10^{-6}$ \\
\hline & $1.01 \cdot 10^{-6}$ & $0.51 \cdot 10^{-6}$ \\
\hline
\end{tabular}

The Wood-Berry binary distillation column separates water and methanol. $Y_{1}$ is the methanol mole fraction in the
TABLE I

PERFORMANCE METRICS FOR THE CLOSED-LOOP WOOD-BERRY SYSTEM USING THE TUNING OBTAINED FROM (26).

distillate [mol\%], $Y_{2}$ is the methanol mole fraction in the bottom product [mol\%], $U_{1}$ is the reflux flow rate $[l b / \mathrm{min}]$, $U_{2}$ is the steam flow rate $[l b / \mathrm{min}]$, and $D$ is the unmeasured feed flow rate $[l b /$ min $]$.

The sampling time of the system is $T_{s}=1[\mathrm{~min}]$. The process and measurement noise covariance for the system are: $R_{w w}=0.0001$ and $R_{v v}=0.0001 \cdot I$. The resulting system is realized as a discrete LTI state space system (15).

The control and prediction horizon for the MPC is selected to $400 \mathrm{~min}$, i.e. $N=400 . A\left(q^{-1}\right)$ and $B\left(q^{-1}\right)$ in (1) are identified such that there is an exact match to $G_{u}(s)$ in (27). Using a robustness bound of $M_{S, \max }=1.775$, the described tuning procedure, i.e. solution of (26), yields the following tuning parameters

$$
\begin{aligned}
& \mathcal{A}=\operatorname{diag}([0.963 ; 0.933]) \\
& Q=\operatorname{diag}([87.3 ; 57.8]) \\
& S=\operatorname{diag}\left(\left[4.87 \cdot 10^{4} ; 6.88 \cdot 10^{4}\right]\right)
\end{aligned}
$$

Table I shows the metrics, $J_{r}$ and $J_{d}$, obtained by solution of (26) for the nominal system with the specified scenarios, $\mathcal{S}$. The corresponding theoretical covariances, $R_{y y}$ and $R_{u u}$, are also illustrated. The simulation-column in Table I shows the metrics, $J_{r}$ and $J_{d}$, for the scenarios simulated with additional process and measurement noise. It is evident that for the chosen tuning, these measures, $J_{r}$ and $J_{d}$, do not deteriorate significantly. The covariances $R_{y y}$ and $R_{u u}$ in the simulation-column of Table I are computed empirically by a Monte Carlo method from a finite sequence of process and measurement noise applied to the closed-loop system without deterministic disturbances and set-point changes. The nice properties of the selected tuning of the controller manifest itself by covariance-matrices having the same size as the covariance matrices for the design case.

We have made an additional simulation using an operating scenario from [12]. The reference for top methanol (distillate) is changed from $96.25[\mathrm{~mol} \%]$ to $97[\mathrm{~mol} \%]$ and at $t=100[\mathrm{~min}]$, a change in the feed occurs. Fig. 2 shows a nominal and a plant-model mismatch simulation for this scenario. For the nominal simulation we assume that $G_{u}(s)$ is exactly known and the model used for the controller design is identical to the model used for simulation. In the mismatch case, the time constants in $G_{u}(s)$ are $75 \%$ of the nominal values for the simulation model. The tuning is robust, since the deviations between the nominal and mismatch case is 

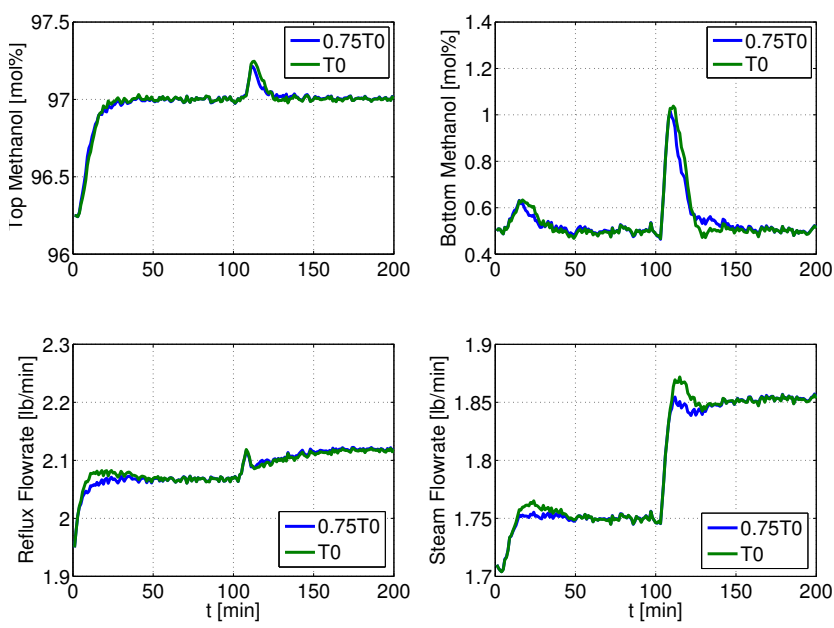

Fig. 2. Nominal and plant-model mismatch simulation of the Wood-Berry distillation column controlled by the ARX based MPC tuned using (26).

marginal. Furthermore, it can be concluded, that the MPC with the selected tuning rejects the disturbance nicely and have good tracking properties.

\section{Cement Mill Circuit}

In this section, we illustrate the tuning procedure and the role of $M_{S, \max }$, for the cement mill system described by [13]. The cement mill is modeled as a continuous-time stochastic input-output model (27) with piecewise constant input signals, i.e. $u(t)=u_{k}, d(t)=d_{k}$ and $w(t)=$ $w_{k} \sim N_{i i d}\left(0, R_{w w}\right)$ in the interval $t_{k} \leq t<t_{k+1}$ and $v\left(t_{k}\right)=v_{k} \sim N_{i i d}\left(0, R_{v v}\right)$. The transfer functions are

$$
\begin{aligned}
& G_{u}(s)=\left[\begin{array}{cc}
\frac{0.62 e^{-5 s}}{(45 s+1)(8 s+1)} & \frac{0.29(8 s+1) e^{-1.5 s}}{(2 s+1)(38 s+1)} \\
\frac{-15 e^{-5 s}}{60 s+1} & \frac{5 e^{-0.1 s}}{(14 s+1)(s+1)}
\end{array}\right] \\
& G_{d}(s)=\left[\begin{array}{c}
\frac{-1.0 e^{-3 s}}{(32 s+1)(21 s+1)} \\
\frac{60}{(30 s+1)(20 s+1)}
\end{array}\right]
\end{aligned}
$$

The variables in the model are: $Y_{1}$ is the elevator load $[k W]$, $Y_{2}$ is the cement fineness $\left[\mathrm{cm}^{2} / \mathrm{g}\right], U_{1}$ is the feed flow rate [TPH], $U_{2}$ is the separator speed [\%], and $D$ is the clinker hardness $[H G I]$.

The continuous-time input-output model (27) is converted to a discrete-time state space model (15) using a sample time of $T_{s}=2[\mathrm{~min}]$. The covariances of the process and measurement noise are: $R_{w w}=1.0$ and $R_{v v}=\operatorname{diag}([0.1 ; 100])$.

The ARX-based MPC is designed for a sampling time of $T_{s}=2[\mathrm{~min}]$ with a prediction and control horizon of $800[\mathrm{~min}]$, i.e. $N=400$. To illustrate the role of the robustness bound, the tuning is performed using two different values for $M_{S, \max }$.

In the first case, we use $M_{S, \max }=1.775$. The tuning parameters obtained by solution of (26) are

$$
\begin{aligned}
\mathcal{A} & =\operatorname{diag}([0.985 ; 0.000]) \\
Q & =\operatorname{diag}([14.1 ; 91.6]) \\
S & =\operatorname{diag}\left(\left[9.68 \cdot 10^{5} ; 9.29 \cdot 10^{3}\right]\right)
\end{aligned}
$$

TABLE II

PERFORMANCE METRICS FOR A CEMENT MILL CONTROLLED BY AN ARX BASED MPC TUNED WITH $M_{S, \max }=1.775$ IN (26).

\begin{tabular}{|c|cc|cc|}
\hline & \multicolumn{2}{|c|}{ Design } & \multicolumn{2}{c|}{ Simulation } \\
\hline \multirow{2}{*}{$J_{r}$} & 81.63 & 2.35 & 212.35 & 176.35 \\
& 0.85 & 7.15 & 3769.40 & 3769.00 \\
\hline \multirow{2}{*}{$J_{d}$} & \multicolumn{2}{|c|}{42.54} & \multicolumn{2}{c|}{184.26} \\
& 22.70 & 0.30 & 0.86 \\
\multirow{2}{*}{$R_{y y}$} & 0.40 & 0.77 & 0.86 & 136.41 \\
\hline \multirow{2}{*}{$R_{u u}$} & 0.77 & 154.16 & 416.46 & -410.12 \\
& 460.01 & -452.10 & -410.12 & 405.50 \\
\hline
\end{tabular}

Table II lists the associated performance metrics for the cement mill controlled by an ARX based MPC using these parameters. The design-column lists the metrics obtained for the nominal system used in selecting the tuning parameters $(\mathcal{A}, Q, S)$. The simulation-column lists the metrics obtained by simulating the closed-loop system with stochastic process and measurement noise using the determined tuning parameters. By inspection of the design-column, $J_{r}, J_{d}$ and $R_{y y}$ look reasonable. The only exception should be the high variance on the cement fineness, $Y_{2}$. However, $R_{u u}$ is very large and suggests that the proposed controller is sensitive to process and measurement noise. This suggestion is confirmed by the simulation-column. When the system is simulated for the scenarios $\mathcal{S}$ with additional process and measurement noise, the integrated absolute error measures, $J_{r}$ and $J_{d}$, deteriorates significantly. The empirically obtained covariances obtained by a stochastic simulation do not change significantly. This illustrates the usefulness of these covariances in assessing the sensitivity of the system. In particular, the sensitivity is often revealed through the magnitude of the input covariances, $R_{u u}$.

To illustrate how useless the proposed tuning with $M_{S, \max }=1.775$ is, the system is simulated for a scenario in which the elevator load is initially changed from $26[k W]$ to $30[k W]$ and a change in the clinker hardness is introduced at $t=800[\mathrm{~min}]$. Fig. 3 illustrates closed-loop simulations for the nominal case and a plant-model mismatch case where the dead times of $G_{u}(s)$ is increased by $50 \%$. In both cases, the tuning gives variances of the input signals that are ridiculous large. Accordingly, the resulting controller is useless in practice.

To improve the robustness of the system and make it less sensitive to noise, the robustness bound is reduced to $M_{S, \max }=1.3$ and a new tuning is computed from (26)

$$
\begin{aligned}
& \mathcal{A}=\operatorname{diag}([0.992 ; 0.852]) \\
& Q=\operatorname{diag}([382 ; 706]) \\
& S=\operatorname{diag}\left(\left[9.91 \cdot 10^{5} ; 4.87 \cdot 10^{5}\right]\right)
\end{aligned}
$$

Table III illustrates the performance metrics of the controlled system with this tuning. From a deterministic point of view, the reduced robustness bound, $M_{S, \max }$, results in worse disturbance rejection and reference tracking. However, the associated input covariance, $R_{u u}$, is significantly lower for the tuning with $M_{S, \max }=1.3$ compared to the tuning with 

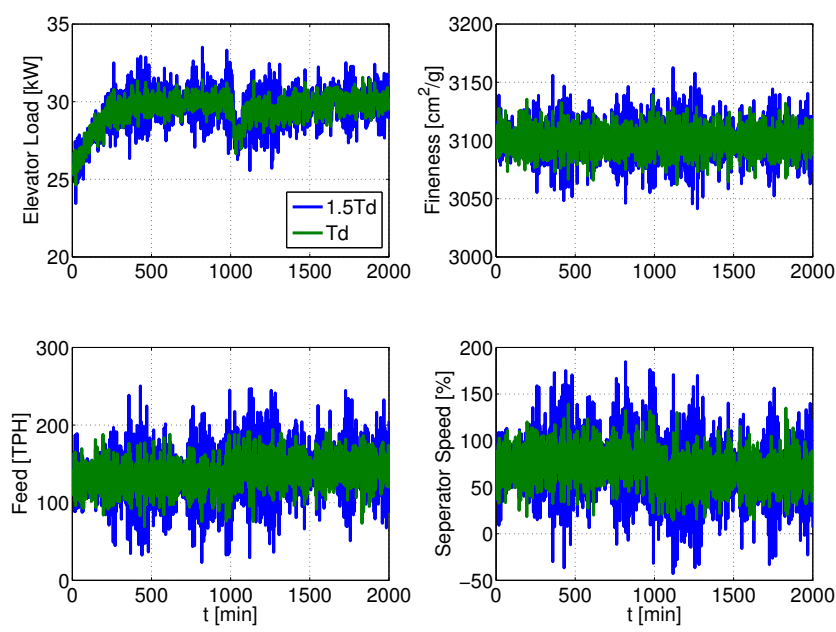

Fig. 3. Nominal and plant-model mismatch simulations of a cement mill controlled by an ARX-based MPC using a tuning with $M_{S, \max }=1.775$.

TABLE III

PERFORMANCE METRICS FOR A CEMENT MILL CONTROLLED BY AN ARX BASED MPC TUNED WITH $M_{S, \max }=1.3 \mathrm{IN}(26)$.

\begin{tabular}{|c|cc|cc|}
\hline & \multicolumn{2}{|c|}{ Design } & \multicolumn{2}{c|}{ Simulation } \\
\hline \multirow{2}{*}{$J_{r}$} & 97.78 & 2.13 & 179.90 & 128.30 \\
& 2.52 & 10.69 & 3245.60 & 3245.50 \\
\hline \multirow{2}{*}{$J_{d}$} & \multicolumn{2}{|c|}{155.49} & \multicolumn{2}{c|}{199.50} \\
& \multicolumn{2}{|c|}{139.75} & \multicolumn{2}{c|}{3277.40} \\
\hline \multirow{2}{*}{$R_{y y}$} & 0.17 & 0.20 & 0.17 & 0.27 \\
& 0.20 & 121.26 & 0.27 & 108.95 \\
\hline \multirow{2}{*}{$R_{u u}$} & 3.45 & -6.29 & 3.19 & -5.77 \\
& -6.29 & 12.24 & -5.77 & 11.18 \\
\hline
\end{tabular}

$M_{S, \max }=1.775$.

The typical working scenario is also simulated for a system using an MPC with this tuning. This simulation is illustrated in Fig. 4. The controller with this tuning is less aggressive. The tracking and disturbance rejection properties are slightly affected, but the system is much less sensitive to stochastic process and measurement noise. By inspection of Fig. 3 and Fig. 4, it is obvious that the tuning obtained with $M_{S, \max }=1.3$ gives the best controller and a controller with acceptable performance. For this controller, the deviations between the nominal and the mismatch case are nearly indistinguishable. This illustrates the nice robustness properties of the controller.

\section{CONCLUSION}

In this paper, we have presented a procedure to tune an ARX-based MPC for multivariate processes. The ARXbased MPC has been designed such that it gives offsetfree control for type I disturbances (steps). The suggested tuning is obtained by minimizing a measure related to the integrated absolute error for a set of pre-determined scenarios. Robustness of the resulting tuning is obtained by restricting the maximum of the sensitivity function by an upper bound. The method has been demonstrated for a binary distillation column and for a cement mill example.

The setup in this paper, using disturbance scenarios, has been used for illustrative purposes. In practice the model
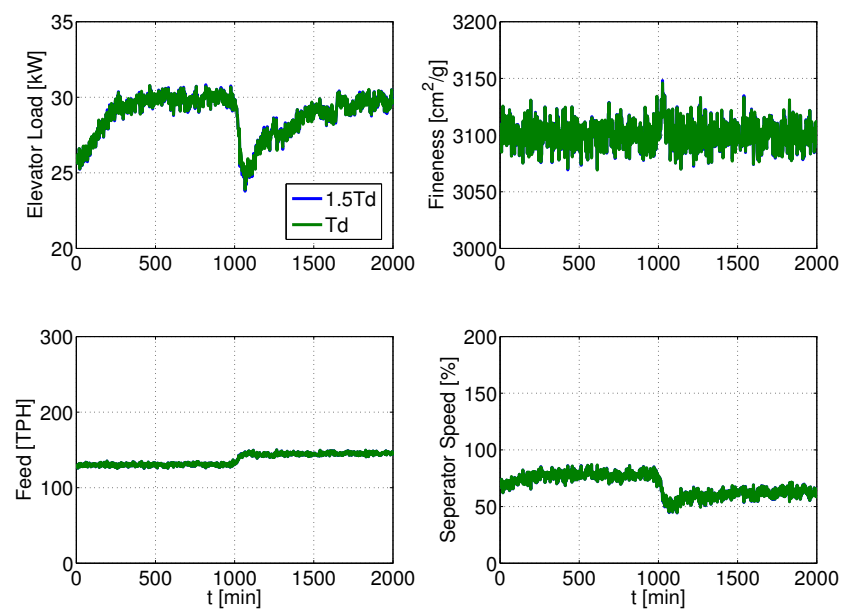

Fig. 4. Nominal and plant-model mismatch simulations of a cement mill controlled by an ARX-based MPC using a tuning with $M_{S, \max }=1.3$.

from the unknown disturbance to the output is not necessarily known. In such cases the disturbance rejection scenarios are replaced by scenarios in which parameters of the ARX model is varied; i.e. the gain, the time delay, or the time constants of the corresponding transfer function model are varied.

\section{REFERENCES}

[1] S. J. Qin and T. A. Badgwell, "A survey of industrial model predictive control technology," Control Engineering Practice, vol. 11, pp. 733764, 2003.

[2] D. A. Finan, J. B. Jørgensen, N. K. Poulsen, and H. Madsen, "Robust model identification applied to type 1 diabetes," in Proceedings of the 2010 American Control Conference, Baltimore, MD, USA, June 30 July 2, 2010 2010, pp. 2021-2026.

[3] J. K. Huusom, N. K. Poulsen, S. B. Jørgensen, and J. B. Jørgensen, "Tuning of methods for offset free MPC based on ARX model representations," in 2010 American Control Conference, 2010, pp. 2355-2360.

[4] —_ "Noise modelling and MPC tuning for systems with infrequent step disturbances," in Proceedings of the 18. IFAC World Congress, 2011, pp. 11226-11232.

[5] J. B. Jørgensen, J. K. Huusom, and J. B. Rawlings, "Finite horizon MPC for systems in innovation form," in 50th IEEE Conference on Decision and Control and European Control Conference, 2011, pp. 1896-1903.

[6] J. K. Huusom, N. K. Poulsen, S. B. Jørgensen, and J. B. Jørgensen, "Tuning SISO offset-free model predictive control based on ARX models," Journal of Process Control, vol. 22, pp. 1997-2007, 2012.

[7] J. L. Garriga and M. Soroush, "Model predictive control tuning methods: A review," Ind. Eng. Chem. Process Design and Developement, vol. 49, pp. 3505-3515, 2010.

[8] J. M. Maciejowski, Predictive Control with Constraints. Prentice Hall, 2002.

[9] R. R. Bitmead and M. Gevers, Adaptive Optimal Control. The Thinking Man's GPC. Prentice Hall International, 1990.

[10] J. H. Lee and Z. H. Yu, "Tuning of model predictive controllers for robust perfomance," Computers in Chemical Engineering, vol. 18, pp. 15-37, 1994.

[11] S. Skogestad and I. Postlethwaithe, Multivariable Feedback Control Analysis and Design. Wiley, 2005.

[12] C. Zhou, J. R. Whiteley, E. A. Misawa, and K. A. M. Gasem, "Application of enhanced LQG/LTR for distillation control," IEEE Control Systems Magazine, vol. 15, pp. 56-63, 1995.

[13] G. Prasath, B. Recke, M. Chidambaram, and J. B. Jørgensen, “Application of soft constrained MPC to a cement mill circuit," in Proceedings of the 9th International Symposium on Dynamics and Control of Process Systems (DYCOPS 2010), 2010, pp. 288-293. 